\title{
A sign assignment in totally twisted Khovanov homology
}

\author{
ANDREW MANION
}

\begin{abstract}
We lift the characteristic-2 totally twisted Khovanov homology of Roberts and Jaeger to a theory with $\mathbb{Z}$ coefficients. The result is a complex computing reduced odd Khovanov homology for knots. This complex is equivalent to a spanning-tree complex whose differential is explicit modulo a sign ambiguity coming from the need to choose a sign assignment in the definition of odd Khovanov homology.
\end{abstract}

$57 \mathrm{M} 25 ; 57 \mathrm{M} 27$

\section{Introduction}

In [4], Lawrence Roberts introduced a "totally twisted" version of $\delta$-graded characteristic-2 Khovanov homology for links. Thomas Jaeger [2] then showed that for knots, the reduced totally twisted Khovanov homology actually coincides with the ordinary reduced Khovanov homology (tensored with a suitable coefficient field). We show how to extend the totally twisted construction over $\mathbb{Z}$, in the context of the odd Khovanov homology of Ozsváth, Rasmussen and Szabó [3]. The result is a chain complex whose homology computes the reduced odd Khovanov homology of knots (again, tensored with a suitable ring). Cancelling some differentials in the complex leads to an equivalent complex whose generators are in bijection with spanning trees of the Tait graph. The coefficient of the differential between two spanning trees is determined up to a sign; the sign ambiguity comes from an analogous ambiguity in odd Khovanov homology, where one must choose a sign assignment on the edges of a cube of resolutions.

\section{Acknowledgements}

I would like to thank John Baldwin, Cotton Seed, Kevin Wilson and my advisor Zoltán Szabó for helpful discussions. I would also like to thank Lawrence Roberts and a referee for comments on a previous version. I was supported by the Department of Defense (DoD) through the National Defense Science and Engineering Graduate Fellowship (NDSEG) Program. This work is also supported by the National Science Foundation Graduate Research Fellowship under grant number DGE 1148900. 


\section{The construction}

We assume the reader is familiar with odd Khovanov homology, as described in [3]. Here we briefly fix notation. Let $D$ be an $n$-crossing diagram for a link $L$, with marks $m_{i}$ assigned to edges. For the general construction, we allow any assignment of marks; to obtain the relationship with spanning trees in Section 4, it will be important that each edge has at least one mark. Let $R$ be the polynomial ring $\mathbb{Z}\left[x_{i}\right]$, with one variable for each mark. Since we will be working with odd Khovanov homology, we also want to choose an orientation for each crossing. Figure 4 below shows one possible choice; the other has the arrow reversed.

Each crossing in $D$ has a 0 -resolution and a 1-resolution. A complete resolution of $D$ gives rise to a diagram with no crossings, which consists of $k$ unlinked circles. To a complete resolution $\rho$, associate the group $V_{\rho}=H^{*}\left(S^{1} \times \cdots \times S^{1}\right)$, where there are $k$ factors of $S^{1}$. This group is actually a ring, and if we label the circles of the resolution $a_{1}, \ldots, a_{k}$, a convenient set of multiplicative generators for $V_{\rho}$ may be labelled $\left\{a_{1}, \ldots, a_{k}\right\}$ as well. The chain complex computing odd Khovanov homology may be written $\left(C_{*}^{\text {odd }}(D), d_{\text {odd }}\right)$, where $C_{*}^{\text {odd }}(D)=\bigoplus_{\rho \in\{0,1\}^{n}} V_{\rho}$. We refer to [3] for the definition of $d_{\text {odd }}$. Here we only remind the reader that while the orientations on the crossings determine split and merge maps with well-defined signs, these naive maps do not automatically fit into a differential satisfying $d_{\text {odd }}^{2}=0$. Rather, one must correct by putting signs on the edges of the cube of resolutions. We will denote the naive maps collectively as $d_{\text {odd }}^{\prime}$ and reserve the name $d_{\text {odd }}$ for the sign-corrected differential.
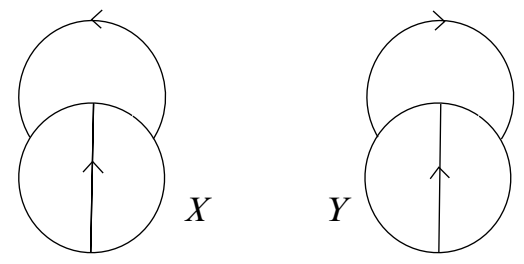

Figure 1: The configurations $X$ and $Y$

The method of correcting the signs makes use of the concept of 2-dimensional (oriented) configurations, which are pictures of a complete resolution along with two (oriented) arcs such that surgery along an arc corresponds to switching a crossing. Two examples of these configurations are depicted in Figure 1, and many more are shown below in Figure 5. In fact, the configurations $X$ and $Y$ in Figure 1 are special; they correspond to faces of the cube of resolutions which both commute and anticommute. The convention we will use is that the configuration labelled $X$ anticommutes and the one labelled $Y$ commutes. We only mention this here because we will need to deal with 2 -dimensional 
configurations in Section 3, and there it will be important that we use this specific convention and not its opposite.

Note that tensoring the odd Khovanov complex with $R$ amounts simply to taking cohomology with $R$ coefficients when defining $V_{\rho}$, and we will use these coefficients from here on.

The construction we discuss amounts to defining a differential $d_{v, \rho}$ on each $V_{\rho}$ (the small $v$ is meant to suggest "vertical," in contrast with the "horizontal" maps of $d_{\text {odd }}$ ). Setting $d_{v}=\sum_{\rho} d_{v, \rho}$, we consider the complex $\left(C_{*}^{\text {odd }}, d_{v}+d_{\text {odd }}\right)$. In the remainder of this section, we will define $d_{v, \rho}$ and show that $\left(d_{v}+d_{\text {odd }}\right)^{2}=0$. When coefficients are taken modulo 2, we will get the complex from [2].

Fix a resolution $\rho$ with circles $a_{1}, \ldots, a_{k}$. The marks $m_{i}$ on $D$ pass to marks on these circles. For each $i$, define $w_{i} \in R$ to be the sum of the variables $x_{j}$ corresponding to those marks $m_{j}$ lying on $a_{i}$. As a preliminary definition, define

$$
d_{v, \rho}^{\prime}=\sum_{i} m\left(w_{i} a_{i}, \cdot\right)
$$

where $m(\cdot, \cdot)$ denotes multiplication in the ring $V_{\rho}$. (Note that the multiplication is anti-commutative, so order matters, and we are multiplying by $w_{i} a_{i}$ on the left.) It is clear that $\left(d_{v, \rho}^{\prime}\right)^{2}=0$, and the same holds for $d_{v}^{\prime}=\sum_{\rho} d_{v, \rho}^{\prime}$. Taking coefficients modulo 2, we get the twisted complex in Jaeger's form [2]: his dot-multiplication maps correspond to our left multiplication.

The definition of $d_{v, \rho}^{\prime}$ is only preliminary since we will need to modify $d_{v, \rho}^{\prime}$ by an overall sign, depending on $\rho$. We now describe this modification. It will be based on the following lemma:

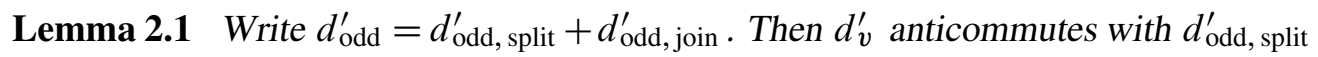
and commutes with $d_{\mathrm{odd}}^{\prime}$, join .

Proof We will do the split case and leave the (very similar) join case to the reader. Consider two resolutions $\rho$ and $\rho^{\prime}$ with a nonzero component of $d_{\text {odd, split between }}^{\prime}$ them. Then $\rho^{\prime}$ is obtained from $\rho$ by splitting one circle $a$ into two circles $b$ and $c$. Choose the labels $b$ and $c$ such that $d_{\text {odd }}^{\prime}(a)=b c$. Denote the passive circles in $\rho$ by $\left\{p_{i}\right\}$; then the remaining circles in $\rho^{\prime}$ may also be labelled $\left\{p_{i}\right\}$.

Homogeneous generators of $V_{\rho}$ take the form $\pi$ or $a \pi$, where here $\pi$ denotes any product of the $p_{i}$. In the following computation, all sums over $q$ indicate sums over 
those passive circles $q$ that are not contained in $\pi$ :

$$
\begin{aligned}
& d_{v}^{\prime} d_{\mathrm{odd}}^{\prime}(\pi)=d_{v}^{\prime}(b-c) \pi=\sum_{q} w_{q} q(b-c) \pi, \\
& d_{\mathrm{odd}}^{\prime} d_{v}^{\prime}(\pi)=d_{\mathrm{odd}}^{\prime}\left(\sum_{q} w_{q} q \pi\right)=\sum_{q} w_{q}(b-c) q \pi=-d_{v}^{\prime} d_{\mathrm{odd}}^{\prime}(\pi) .
\end{aligned}
$$

Similarly,

$$
\begin{aligned}
d_{v}^{\prime} d_{\mathrm{odd}}^{\prime}(a \pi) & =d_{v}^{\prime}(b c \pi)=\sum_{q} w_{q} q b c \pi \\
d_{\mathrm{odd}}^{\prime} d_{v}^{\prime}(a \pi) & =d_{\mathrm{odd}}^{\prime}\left(\sum_{q} w_{q} q a \pi\right)=-d_{\mathrm{odd}}^{\prime}\left(\sum_{q} w_{q} a q \pi\right) \\
& =-\sum_{q} w_{q} b c q \pi=-\sum_{q} w_{q} q b c \pi=-d_{v}^{\prime} d_{\mathrm{odd}}^{\prime}(a \pi) .
\end{aligned}
$$

The preceding lemma tells us that, to properly define $d_{v}$, we should flip the signs on $d_{v, \rho}^{\prime}$ for some vertices $\rho$ of the cube of resolutions. For each split edge of the cube, we want the endpoints to receive the same sign-change, while for each join edge, we want the endpoints to receive the opposite sign-change. As with odd Khovanov homology, a cohomological argument allows us to make these choices:

Proposition 2.2 It is possible to flip the signs on some of the $d_{v, \rho}^{\prime}$ such that the above conditions hold, and the flips are unique up to an overall sign.

Proof Consider the usual CW structure of the cube $Q=[0,1]^{n}$ of resolutions, with the $k$-skeleton consisting of the $k$-dimensional faces. Define a cochain $\tau \in C^{1}(Q ; \mathbb{Z} / 2 \mathbb{Z})$ by labelling split edges 0 and join edges 1 . We want to show that $\tau$ is a coboundary, but since $Q$ is contractible, it suffices to show $\tau$ is a cocycle. To compute $\delta \tau$, we look at the 2-dimensional faces of $Q$. There are five types of these, corresponding to the five possible unoriented 2-dimensional configurations. These are shown in Figure 2. For each of these five configurations, the sum of $\tau$ along the boundary edges of the corresponding 2 -dimensional face is $0(\bmod 2)$; this can easily be checked. Hence $\delta \tau=0$, so $\tau=\delta \sigma$ for some $\sigma \in C^{0}(Q ; \mathbb{Z} / 2 \mathbb{Z})$. Flip the sign on a vertex $\rho$ if $\sigma(\rho)=1$, and leave it alone if $\sigma(\rho)=0$. Note that $\sigma$ is unique up to an overall sign since $H^{0}(Q ; \mathbb{Z} / 2 \mathbb{Z})=\mathbb{Z} / 2 \mathbb{Z}$.

Remark 2.3 For the purpose of computation, an explicit formula for $\sigma$ can be given by

$$
\sigma(\rho)=\frac{1}{2}\left(|\rho|-k(\rho)+k\left(\rho_{0}\right)\right) \bmod 2,
$$




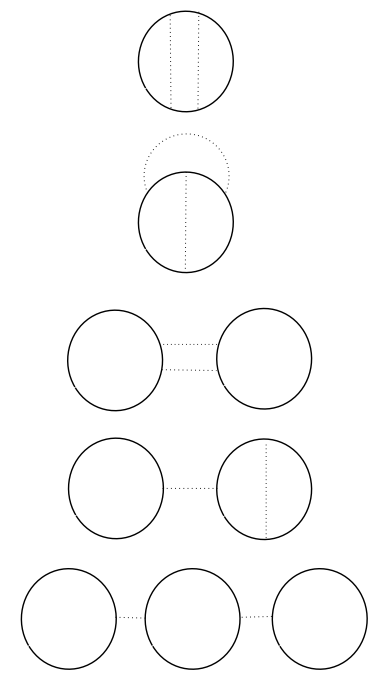

Figure 2: The five types of unoriented 2-dimensional configurations. Dotted lines indicate the arcs of the each configuration.

where $\rho$ is a vertex of the cube of resolutions, $|\rho|$ is the number of 1-resolutions chosen at crossings in $\rho$, and $k(\rho)$ is the number of closed circles in $\rho$. The resolution $\rho_{0}$ is the "all zeroes" resolution.

Indeed, for two $\rho$ differing in a crossing change, clearly $\sigma$ has the same value on both resolutions if the crossing change is a split, since $|\rho|$ increases by one while $-k(\rho)$ decreases by one. Similarly, for a join, both $|\rho|$ and $-k(\rho)$ increase by one, so $\sigma(\rho)$ changes value.

By the proof above, the only ambiguity in this formula for $\sigma$ is an overall sign flip; in other words, we could equally well add a +1 to the formula if we wanted. The above formula is normalized so that $\sigma\left(\rho_{0}\right)=0$.

If we define $d_{v}$ by making the appropriate sign flips, then $d_{v}$ anticommutes with $d_{\text {odd }}^{\prime}$, and of course $d_{v}^{2}=0$. Finally, correct the signs in $d_{\text {odd }}^{\prime}$ as in standard odd Khovanov homology (using another cohomological argument; see [3]). It is still true that $d_{v}$ anticommutes with $d_{\text {odd }}$, so we have $\left(d_{v}+d_{\text {odd }}\right)^{2}=0$. This completes the construction of a complex that reduces to Jaeger's twisted complex modulo 2.

Remark 2.4 If we have a basepoint on our link, we can define reduced versions of everything above in the standard way. 


\subsection{Invariance}

The homotopy invariance of ( $\left.C_{*}^{\text {odd }}, d_{v}+d_{\text {odd }}\right)$ under the Reidemeister moves can be proved using an argument of Baldwin, similar to that used in [1]. For R1 and R2, one writes down the complexes before and after the move, and then cancels differentials in the "before" complex to obtain the "after" complex. Once invariance under R2 is proven, invariance under R3 amounts to considering the braid word $x y x y^{-1} x^{-1} y^{-1}$, where $x$ and $y$ are elementary 3-strand braid group generators, and showing its appearance in a Khovanov complex is equivalent to the identity. The relevant "before" complex comes from a 64-vertex cube of resolutions which was dealt with in [1]. In our case, the presence of $d_{v}$ makes things a bit more complicated, a priori. Luckily, we can use Theorem 3.1 to remove the complications. In this section we will briefly sketch the proof for invariance under R1, and see how using Theorem 3.1 makes things easier.

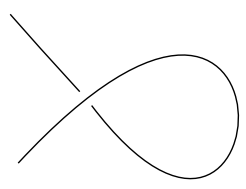

Before R1

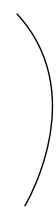

After R1

Figure 3: The R1 move

Consider an R1 move which undoes a positive kink; see Figure 3. Write $C_{*}^{\text {before }}$ for the complex before undoing the kink and $C_{*}^{\text {after }}$ for the complex after performing R1. Without regard to the differential, $C_{*}^{\text {before }}$ is the sum of three pieces: $C_{*}^{\text {before }}=$ $C_{0,+} \oplus C_{0,-} \oplus C_{1}$, where the 0 or 1 indicates the resolution at the crossing in question and the + (resp. - ) denotes those generators represented by monomials not containing (resp. containing) the isolated small circle in the 0 -resolution. In each of the three local pictures in question, label the non-closed component as $a$ and the isolated small circle (in the 0 -pictures) as $b$. Denote the differential from $C_{0, \pm}$ to $C_{1}$ by $d_{ \pm}$.

In fact, $d_{+}$maps generators of $C_{0,+}$ bijectively to generators of $C_{1}$. We would like to cancel all components of $d_{+}$, leaving ourselves with $C_{0,-}$ and an induced differential on this summand. There are some obvious components of this differential, namely those coming from components internal to $C_{0,-}$ in the whole complex $C_{*}^{\text {before }}$. These components correspond to almost all of the differential on $C_{*}^{\text {after }}$ under the bijection sending a generator $p$ of $C_{*}^{\text {after }}$ to $b p$ in $C_{0,-}$. The only things missing are the vertical differentials from marks on $b$. We want to show that the induced differential from cancellation precisely adds in these missing components. 
Indeed, any induced components come from compositions

$$
C_{0,-} \stackrel{d_{-}}{\longrightarrow} C_{1} \stackrel{d_{+}^{-1}}{\longrightarrow} C_{0,+} \stackrel{d_{v, b}}{\longrightarrow} C_{0,-},
$$

where the final map comes from the component of $d_{v}$ associated to marks on $b$. The map $d_{-}$(a join) is nonzero only on elements of the form $b p$, where $p$ does not contain $a$. We have $d_{-}(b p)= \pm a p$, but applying $d_{+}^{-1}$, the sign cancels and we get $a p \in C_{0,+}$. Applying $d_{v, b}$ gives us $w \cdot b a p$, where $w$ is the sum of the weights of marks on $b$. This was precisely the component we were looking for, and invariance under R1 follows.

Note that if there are no marks on $b$, then there is also no induced differential from cancellation. Thus, if we first used Theorem 3.1 to move all marks off the edges comprising $b$, then the proof of $R 1$ invariance would be exactly the same as in [1]. The same holds for $R 2$ and $R 3$ invariance; one just needs to move all marks off complete "internal" circles appearing in the relevant diagrams. The proofs would be considerably more complicated without moving the marks first (the author would like to thank Lawrence Roberts for pointing this out).

\section{Relation with odd Khovanov homology}

Jaeger shows that for knots, his (reduced) complex actually computes reduced Khovanov homology. We would like to do the same with reduced odd Khovanov homology. Suppose $L$ is actually a knot $K$ with basepoint $p$. Following [2], the main point is that we can move marks past crossings without changing the isomorphism type of the twisted complex. Consider a mark $m$ near a crossing $c$ of $D$, with local picture $x$. Let $D^{\prime}$ be the marked diagram with this local picture replaced by $y_{x}$. We may assume that $c$ is oriented as in Figure 4 and that the crossing orientations on $D^{\prime}$ are the same as on $D$.

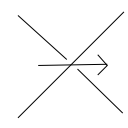

Figure 4: Orientation at the crossing $c$

Theorem 3.1 The twisted complexes associated to $D$ and to $D^{\prime}$ are isomorphic.

An analogous statement holds when sliding a mark over a crossing, rather than under. As in [2], Theorem 3.1 (plus the analogous statement) immediately implies that the reduced twisted complex computes reduced odd Khovanov homology for knots. (More precisely, it computes Khovanov homology tensored with $\mathbb{Z}\left[x_{i}\right]$ ). Indeed, one can 
simply move all the marks to the same edge as the basepoint, effectively killing $d_{v}$ and leaving only $d_{\text {odd }}$ in the differential. We will now prove Theorem 3.1.

Proof In [2], with coefficients taken modulo 2, this theorem is a purely local computation. Unfortunately, in odd Khovanov homology, signs on maps are not determined entirely by local data, so we must work a bit harder. Still, Jaeger's chain map (with appropriate signs) will work in our situation.

To define the map, it will be convenient to follow [2] and use local pictures. In this notation, the complex of $D$ will be written $)(\oplus \asymp$, where each summand actually represents all summands of the total complex of $D$ whose resolution at $c$ is as depicted. There is one such summand for each "outer" resolution, ie, each resolution $\rho$ of all crossings except $c$. The complex of $D^{\prime}$ will also be written as $)(\oplus \asymp$, with the same interpretation.

Fix an outer resolution $\rho$. With respect to the summands $)($ and $\asymp$, the isomorphism of complexes is given by

$$
\left.F_{\rho}:=\left(\begin{array}{rr}
x & \pm \asymp \\
& \asymp
\end{array}\right):\right)(\oplus \asymp \rightarrow)(\oplus \asymp .
$$

The sign in this formula will depend on $\rho$ in a way that will be specified below. Regardless of the chosen sign, it is clear that each $F_{\rho}$ is invertible; the inverse is the same map with opposite sign. Set $F=\sum_{\rho} F_{\rho}$. Then $F$ is invertible, and we only need show that $F$ commutes with $d_{v}$ and $d_{\text {odd }}$. We may write $F_{\rho}=\mathrm{id}+H_{\rho}$, and this will be useful later.

In order to specify the sign on $H_{\rho}$, consider $\left(C_{*}^{\text {odd }}(D), d_{\text {odd }}\right)$, which is the same as $\left(C_{*}^{\text {odd }}\left(D^{\prime}\right), d_{\text {odd }}\right)$ since the only difference between $D$ and $D^{\prime}$ is the placement of a mark. There is a component $d_{c, \rho}$ of $d_{\text {odd }}$ coming from $c$. It has a naive sign from the orientation on $c$; write $\sigma\left(d_{c, \rho}\right)=0$ if the actual sign agrees with the naive sign and $\sigma\left(d_{c, \rho}\right)=1$ otherwise. In the process of defining $d_{v}$ above, we also put signs on certain vertices of the complete cube of resolutions. There are two such vertices associated to $\rho$; call them $(\rho, 0)$ and $(\rho, 1)$ where the 0 or 1 denotes the resolution of $c$. Write $\sigma(\rho, i)=0$ if we did not flip the sign on $d_{v,(\rho, i)}$, and write $\sigma(\rho, i)=1$ if we did. Define the sign on $H_{\rho}$ to be

$$
\sigma\left(H_{\rho}\right):=\sigma\left(d_{c, \rho}\right) \cdot \sigma(\rho, 1) .
$$

To show $F$ is a chain map, we will first consider those components of $d_{v}$ and $d_{\text {odd }}$ which correspond to the mark $m$ and the crossing in the local picture $\times($ or $1 / x$ ). These are the components Jaeger deals with in [2]. The relevant commutative diagram in his 
paper also works in our situation, once it is suitably interpreted, and once signs are added.

$$
\begin{aligned}
& )\left(\oplus \asymp \stackrel{\left(\begin{array}{cc}
w)( & \\
)( & w \asymp
\end{array}\right)}{\longrightarrow}\right)(\oplus \asymp
\end{aligned}
$$

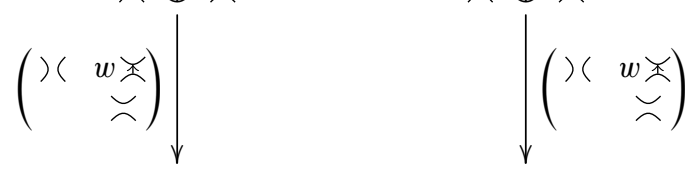

$$
\begin{aligned}
& \left.x \oplus \asymp \underset{\left(\begin{array}{cc}
w)< & \\
) \times( & w \curvearrowleft
\end{array}\right)}{\longrightarrow}\right)(\oplus \asymp
\end{aligned}
$$

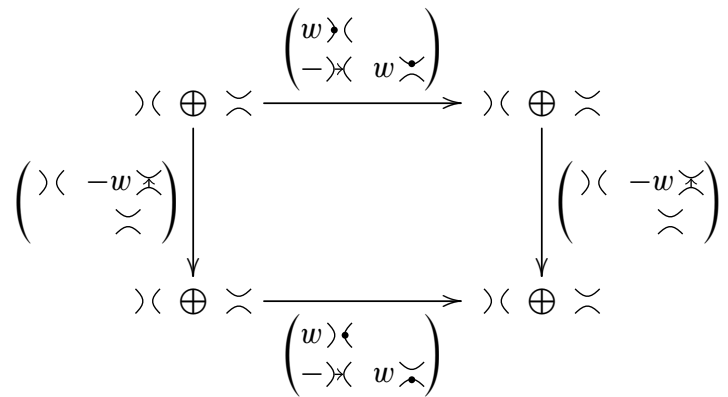

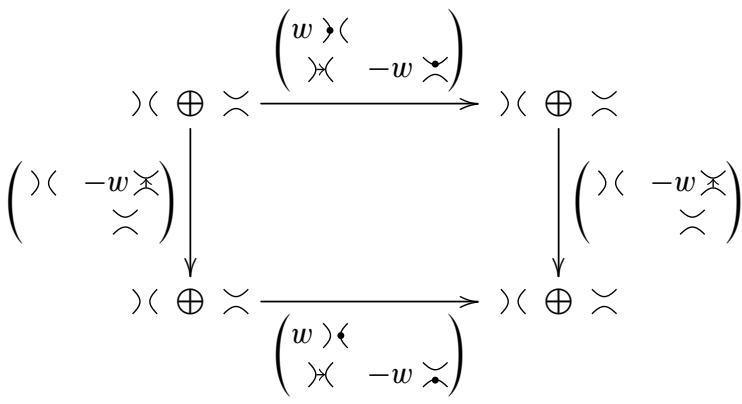

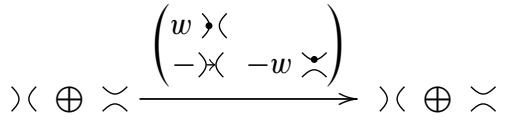

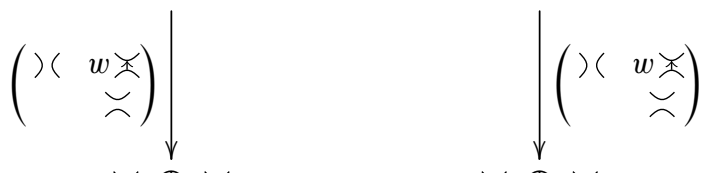

$$
\begin{aligned}
& \left.x \oplus \frac{\left(\begin{array}{l}
w)< \\
-) \times(-w \asymp)
\end{array}\right)}{\longrightarrow}\right)(\oplus \asymp
\end{aligned}
$$


Above are four copies of the diagram from [2] with signs added. They cover the possible cases when $\sigma(\rho, 0)=0$ (in other words, all cases in which the upper-left entries of the horizontal maps have a + sign). It turns out that, once we consider the case $\sigma(\rho, 0)=0$, the case $\sigma(\rho, 0)=1$ involves the same set of matrix multiplications, up to an overall sign. The diagrams on the left have $\sigma\left(d_{c, \rho}\right)=0$ and the ones on the right have $\sigma\left(d_{c, \rho}\right)=1$. In the top diagrams, $\sigma(\rho, 1)=0$ (so the crossing change at $c$ is a split), and in the bottom ones, $\sigma(\rho, 1)=1$ (so the crossing change is a join). On the horizontal maps, a dot represents left multiplication by whichever circle contains the dot. The vertical maps $\left(H_{\rho}\right)$ have signs as specified in Equation (1).

The reader may check, by multiplying matrices, that the diagrams above do commute. A few relations will be needed. First, $) \times(x=\asymp-\asymp$, and this holds regardless of whether the crossing change is a split or a join. (One sees this by explicitly writing down the maps when the first cobordism is a split and when it is a join.) Analogously, ×0 $)(x=)(-)<$.

There are also relations depending on whether the crossing change at $c$ is a split (as in the top two diagrams) or a join (as in the bottom two diagrams). When the crossing change is a split, we have $)<0 \asymp=\asymp 0 \succsim$, as well as $\rangle(=)<$ since the dots are on the same circle. When it is a join, we have the relations $) \prec 0 \asymp=-\asymp 0 \asymp$ and $\asymp=\asymp$. With these relations, one can see that the four diagrams above do commute. There are four more diagrams to consider, with $\sigma(\rho, 0)=1$, but these computations follow from the same set of matrix multiplications. The only difference is that some matrices pick up an overall factor of -1 .

Next we want to show that $F$ commutes with those components of $d_{v}$ corresponding to marks outside the local crossing picture. We may fix an outer resolution $\rho$. Writing $F_{\rho}=\mathrm{id}+H_{\rho}$, we can restrict attention to $H_{\rho}$, because id commutes with everything outside the local picture. There are several cases to consider, and each is an easy algebraic computation. We will consider the cases when the crossing change at $c$ is a join; the split case is very similar.

First of all, note that since $\rho$ is fixed, it does not matter here which sign was assigned to $H_{\rho}$, so we may assume the sign is positive.

The crossing change at $c$ is a join or a split. Assuming it is a join, by our earlier construction, we applied a sign change either to $d_{v}^{\prime}$ before the crossing change or after (not both); in other words, $\sigma(\rho, 0) \neq \sigma(\rho, 1)$. Hence we want to show that $H_{\rho}$ anticommutes with the relevant components of $d_{v}^{\prime}$ (recall that the ' indicates the naive signs). The domain of $H_{\rho}$ is the resolution $(\rho, 1)$. This has one circle, say $a$, which intersects the local crossing picture, and possibly several other circles (say $\left\{p_{i}\right\}$ ). $H_{\rho}$ splits $a$ into two circles, say $b$ and $c$ (chosen so that $\left.H_{\rho}(a)=b c\right)$. Let $\pi$ be a 
monomial in the $p_{i}$. Let $m$ be a mark outside the local picture; $m$ may lie on any circle $q$ of the resolution (possibly $a$ ). Let $w$ denote the variable associated to $m$, and let $d_{v, q}^{\prime}$ be the component of $d_{v}^{\prime}$ coming from $q$.

If $q$ is not $a$, then

$$
d_{v, q}^{\prime}\left(H_{\rho}(\pi)\right)=d_{v, q}^{\prime}((b-c) \pi)=w q(b-c) \pi,
$$

while

$$
H_{\rho}\left(d_{v, q}^{\prime}(\pi)\right)=H_{\rho}(w q \pi)=w(b-c) q \pi,
$$

which equals $-d_{v, q}^{\prime}\left(H_{\rho}(\pi)\right)$ as desired. Similarly,

$$
d_{v, q}^{\prime}\left(H_{\rho}(a \pi)\right)=d_{v, q}^{\prime}(b c \pi)=w q b c \pi,
$$

while

$$
H_{\rho}\left(d_{v, q}^{\prime}(a \pi)\right)=H_{\rho}(w q a \pi)=-H_{\rho}(w a q \pi)=-w b c q \pi,
$$

which is $-d_{v, q}^{\prime}\left(H_{\rho}(a \pi)\right)$ as desired.

Now, suppose $q=a$ and the mark $m$ lies on $b$ after the split. We have

$$
d_{v, q}^{\prime}\left(H_{\rho}(\pi)\right)=d_{v, q}^{\prime}((b-c) \pi)=-w b c \pi,
$$

while

$$
H_{\rho}\left(d_{v, q}^{\prime}\right)(\pi)=H_{\rho}(w a \pi)=w b c \pi,
$$

which is $-d_{v, q}^{\prime}\left(H_{\rho}(\pi)\right)$. For $a \pi$, the composition either way gives zero. If the mark instead lies on $c$ after the split, the computation is exactly analogous, so we have finished with the case where the crossing change at $c$ is a join.

The split case is very similar. We may assume no sign changes were applied to $d_{v}^{\prime}$ before or after the crossing; since $\sigma(\rho, 0)=\sigma(\rho, 1)$, we may assume both are zero. We now want to show $H$ commutes with $d_{v}^{\prime}$. We must consider marks on the top local circle, on the bottom local circle, and disjoint from either circle. After doing the computations in each case, we see that $H$ commutes with $d_{v}$.

Our final task is to show $F$ (or equivalently $H$ ) commutes with $d_{\text {odd }}$, and we need only consider components of $d_{\text {odd }}$ coming from crossings outside our local picture. Consider an external crossing $e$, and fix a resolution of all the other external crossings. We get two outer resolutions $\rho$ and $\rho^{\prime}$, where in $\rho$ we take the 0 -resolution at $e$ and in $\rho^{\prime}$ we take the 1 -resolution. Let $\Delta \sigma(H)=\sigma\left(H_{\rho}\right)-\sigma\left(H_{\rho^{\prime}}\right)$ (taken modulo 2). Let $\Delta \sigma(e)=\sigma\left(d_{e, \rho}\right)-\sigma\left(d_{e, \rho^{\prime}}\right)$ with notation similar to above. We may define $\Delta \sigma(c)$ analogously. 
Next consider the two-dimensional configuration generated by $c$ and $e$ (with all other crossings resolved as we decided above). Call this $f$ the "forward" configuration. There is a square associated to $f$; its sides come from $d_{c}^{\prime}$ and $d_{e}^{\prime}$ (without sign corrections). Write $a_{f}=0$ if it commutes and $a_{f}=1$ if it anti-commutes; if it does both, use the convention specified in Section 2 (ie, the configuration $X$ anticommutes and the configuration $Y$ commutes, and these are the only two ambiguous configurations).

From $f$ we can obtain a "backward" configuration by resolving $c$ and replacing the corresponding oriented arc by one rotated 90 degrees counter-clockwise. Call this configuration $b$. Figure 5 has many examples of configurations and their backwards partners. For the backward configuration, we again get a square that either commutes (set $a_{b}=0$ ) or anti-commutes (set $a_{b}=1$ ). The sides of this square come from $d_{e}^{\prime}$ and $H$ (taken with the "naive" positive sign), since $H$ amounts to doing the crossing change at $c$ "backwards."

What we want to show is that $\Delta \sigma(H)+\Delta \sigma(e)+a_{b}=0$ modulo 2. The following lemma allows us to do this:

Lemma 3.2 For a two-dimensional configuration associated to oriented crossings $c$ and $e$ in a diagram, define $f, b, a_{f}$ and $a_{b}$ as above ( $a_{f}$ and $a_{b}$ have values mod 2).

(1) If the arc associated to $e$ is a split in the backward configuration $b$, then $a_{f}=$ $a_{b}+1$.

(2) If it is a join, then $a_{f}=a_{b}$.

Proof There are several cases to be considered; in fact, a diagram is more useful than words here. Figure 5 depicts the relevant 2-dimensional configurations. The left column shows the forward and backward configurations such that $e$ is a split in the backwards configuration. The right column does the same for configurations where $e$ is a join in the backwards configuration. All configurations are labeled with "comm." if the corresponding square commutes and "anti." if it does not. The content of the lemma is that these labels are correct (each is a simple verification), plus the fact that in the left column, a configuration and its backwards partner have opposite labels while in the right column they have the same labels. Note that our choice of convention for the configurations $X$ and $Y$ of Figure 1 is needed for the lemma to hold.

Lemma 3.2 immediately finishes the proof of Theorem 3.1. Indeed, we know $\Delta \sigma(e)+$ $\Delta \sigma(c)+a_{f}=1$ modulo 2, since odd Khovanov homology satisfies $d^{2}=0$. Note that $\Delta \sigma(H)=\Delta \sigma(c)$ when we are in the first case of Lemma 3.2, and $\Delta \sigma(H)=\Delta \sigma(c)+1$ otherwise. Hence in either case we can conclude $\Delta \sigma(H)+\Delta \sigma(e)+a_{b}=0$, as desired. 


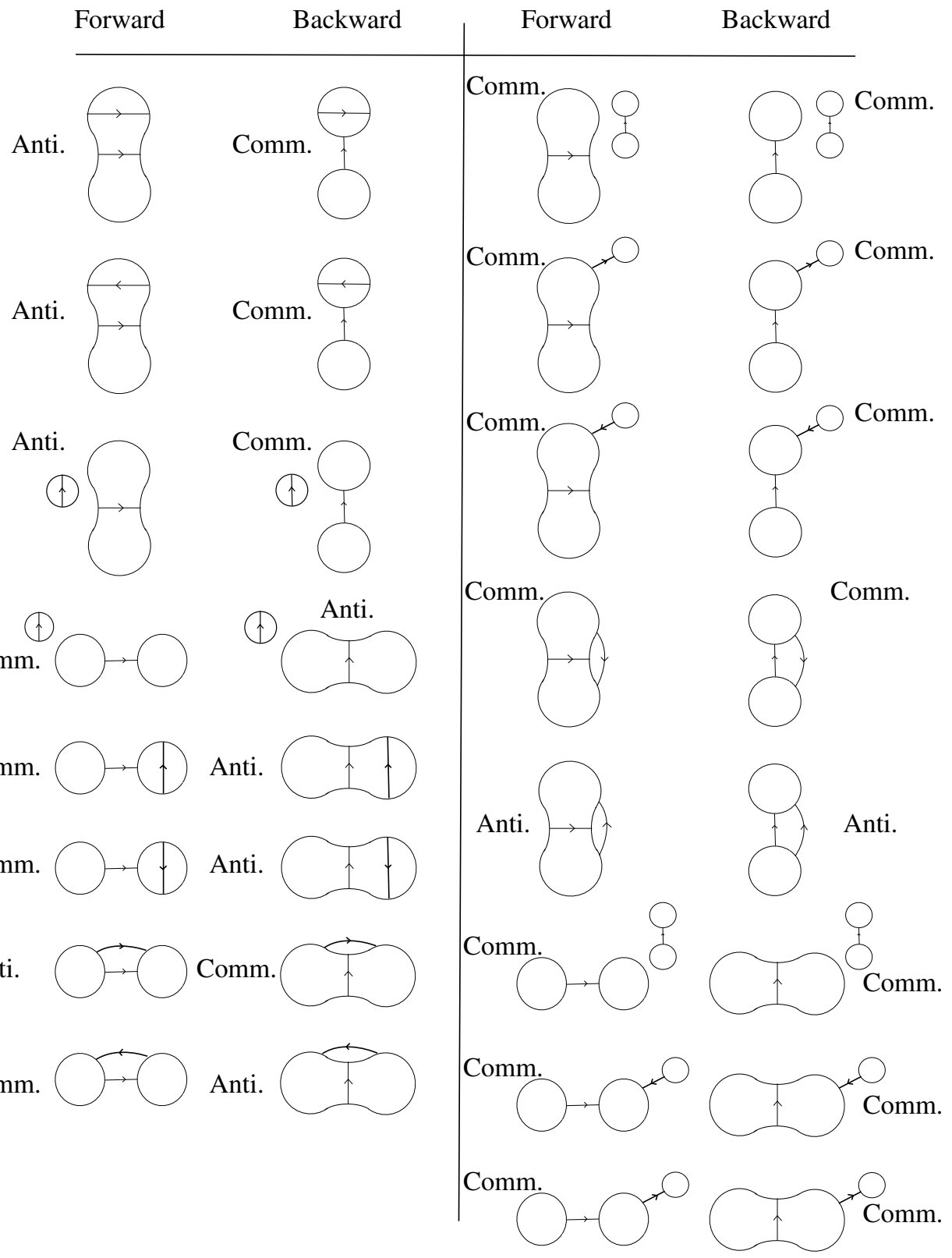

Figure 5: The cases needed for Lemma 3.2 


\section{Spanning trees}

As in [2] and [4], after inverting some of $R=\mathbb{Z}\left[x_{i}\right]$, one can cancel the vertical differentials in the reduced twisted complex to obtain a spanning-tree complex computing reduced odd Khovanov homology. Things will not be quite as nice as in characteristic 2 , since the lack of a way to canonically determine signs in odd Khovanov homology will lead to a sign ambiguity in the spanning-tree differential. Still, we will discuss the situation briefly.

Let $S$ denote the ring obtained from $R$ by inverting all products of sums of the form $x_{i_{1}}+\cdots+x_{i_{l}}$, where $i_{1}, \ldots, i_{l}$ index some subset of the marks. Form the twisted complex with coefficients in $S$. Consider a complete resolution $\rho$, with circles $a_{1}, \ldots, a_{k}$. Let $w_{i}$ denote the sum of the variables corresponding to marks on $a_{i}$. The complex $\left(V_{\rho}, d_{v, \rho}\right)$ is actually the Koszul complex associated to the elements $w_{1}, \ldots, w_{k}$ of $S$. We want to show this complex is acyclic.

In fact, the Koszul complex would already be acyclic over $R$, except in the lowest degree (since the $w_{i}$ form a regular sequence in $\mathbb{Z}\left[x_{i}\right]$ ). After tensoring with $S$, all the $w_{i}$ become invertible, and the lowest homology of the complex (namely $S$ modulo the $\left.w_{i}\right)$ is also trivial.

So after cancellation of $d_{v}$, we get a complex where the only contributions come from connected resolutions, ie, spanning trees of the Tait graph of $D$. Consider two connected resolutions $T$ and $\widetilde{T}$, differing at only two crossings. There are two intermediate two-component resolutions $\rho$ and $\rho^{\prime}$ between them. In each, one circle contains the basepoint. Let $w$ (resp. $w^{\prime}$ ) denote the sum of the variables of the marks on the circle without the basepoint in $\rho$ (resp. $\rho^{\prime}$ ). Then the contribution to $\partial T$ in the spanning-tree complex, if we used the naive maps, would be the sum of

$$
T \rightarrow \rho \stackrel{1 / w}{\longrightarrow} \rho \rightarrow \widetilde{T} \text { and } T \rightarrow \rho^{\prime} \stackrel{1 / w^{\prime}}{\longrightarrow} \rho^{\prime} \rightarrow \widetilde{T} .
$$

To put in the actual signs, note that both $\rho$ and $\rho^{\prime}$ come from splitting circles in $T$. Hence the sign-corrections to $d_{v, \rho}$ and $d_{v, \rho^{\prime}}$, and hence to $1 / w$ and $1 / w^{\prime}$, are the same. The other four maps, though, need to form an anticommuting square in the cube of resolutions. The maps come from a 2-dimensional configuration that is either $X$ or $Y$, depending on the orientations of the relevant crossings. Recall that our convention was that $X$ anticommutes and $Y$ commutes. Hence if the configuration is $Y$, one or three of the four maps must pick up a sign. Because of this sign, the coefficient of the differential from $T$ to $\widetilde{T}$ is $\pm\left(1 / w-1 / w^{\prime}\right)$. On the other hand, if the configuration is $X$, the coefficient from $T$ to $\widetilde{T}$ is $\pm\left(1 / w+1 / w^{\prime}\right)$. The author does not know a good way to decide between the + and - signs on the outside without actually making explicit sign assignments in the cube of resolutions. 


\section{References}

[1] J A Baldwin, On the spectral sequence from Khovanov homology to Heegaard Floer homology, Int. Math. Res. Not. 2011 (2011) 3426-3470 MR2822178

[2] T C Jaeger, A remark on Roberts' totally twisted Khovanov homology, J. Knot Theory Ramifications 22 (2013) 1350022, 16 MR3070834

[3] PS Ozsváth, J Rasmussen, Z Szabó, Odd Khovanov homology, Algebr. Geom. Topol. 13 (2013) 1465-1488 MR3071132

[4] L Roberts, Totally twisted Khovanov homology arXiv:1109.0508

Department of Mathematics, Princeton University

Princeton, NJ 08544, USA

amanion@math.princeton.edu

Received: 9 June 2012 Revised: 16 September 2013 